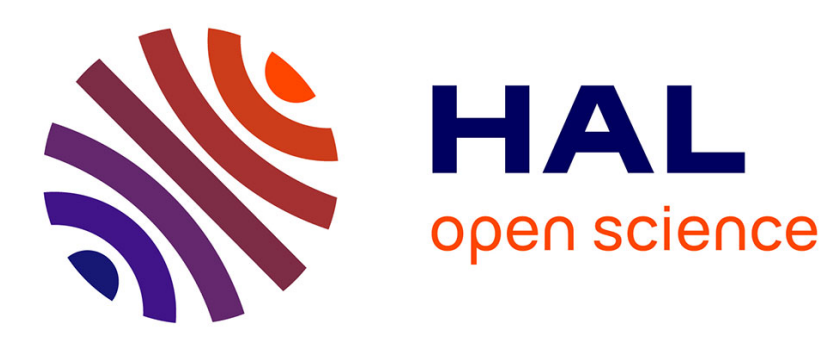

\title{
Programming the Emergence in Morphogenetically Architected Complex Systems
}

Franck Varenne, Pierre Chaigneau, Jean Petitot, René Doursat

\section{To cite this version:}

Franck Varenne, Pierre Chaigneau, Jean Petitot, René Doursat. Programming the Emergence in Morphogenetically Architected Complex Systems. Acta Biotheoretica, 2015, 63 (3), pp.295 - 308. 10.1007/s10441-015-9262-z . hal-01516255

\section{HAL Id: hal-01516255 \\ https://hal.science/hal-01516255}

Submitted on 29 Apr 2017

HAL is a multi-disciplinary open access archive for the deposit and dissemination of scientific research documents, whether they are published or not. The documents may come from teaching and research institutions in France or abroad, or from public or private research centers.
L'archive ouverte pluridisciplinaire HAL, est destinée au dépôt et à la diffusion de documents scientifiques de niveau recherche, publiés ou non, émanant des établissements d'enseignement et de recherche français ou étrangers, des laboratoires publics ou privés. 


\title{
Programming the Emergence in Morphogenetically Architected Complex Systems
}

\author{
Franck Varenne • Pierre Chaigneau • \\ Jean Petitot · René Doursat
}

\begin{abstract}
Large sets of elements interacting locally and producing specific architectures reliably form a category that transcends the usual dividing line between biological and engineered systems. We propose to call them morphogenetically architected complex systems (MACS). While taking the emergence of properties seriously, the notion of MACS enables at the same time the design (or "meta-design") of operational means that allow controlling and even, paradoxically, programming this emergence. To demonstrate our claim, we first show that among all the self-organized systems studied in the field of Artificial Life, the specificity of MACS essentially lies in the close relation between their emergent properties and functional properties. Second, we argue that to be a MACS a system does not need to display more than weak emergent properties. Third, since the notion of weak emergence is based on the possibility of simulation, whether computational or mechanistic via machines, we see MACS as good candidates to help design artificial self-architected systems (such as robotic swarms) but also harness and redesign living ones (such as synthetic bacterial films).
\end{abstract}

Keywords Artificial life · Bio-inspiration - Complex systems · Self-architected systems · Functional form - Weak emergence · Pattern formation · Morphogenesis · Morphogenetic engineering $\cdot$ Swarm robotics $\cdot$ Synthetic biology

Franck Varenne

Sorbonne Research Group in Sociological Analysis Methods (GEMASS), CNRS (UMR8598), ParisSorbonne University, France; and University of Rouen, France - E-mail: franck.varenne@ univ-rouen.fr

Pierre Chaigneau

"Science, Philosophy, History" (SPHERE) Laboratory, CNRS (UMR7219), University Paris-Diderot (Paris 7), France - E-mail: pichaigneau@laposte.net

Jean Petitot

Center for Social Analysis and Mathematics (CAMS), CNRS (UMR8557), School of Advanced Studies in Social Sciences (EHESS), Paris, France - E-mail: jean.petitot@ehess.fr

René Doursat

Complex Systems Institute Paris Ile-de-France (ISC-PIF), CNRS (UPS3611), Paris, France; and BioEmergences Laboratory, CNRS (UPS3674), Gif-sur-Yvette, France - E-mail: rene.doursat@iscpif.fr *corresponding author 


\section{Introduction}

Engineering design and control are traditionally not associated with "natural science", whether looking at geophysical or biological phenomena. By definition, the latter happen spontaneously (climate, evolution), outside any planning or intervention (only perturbed by the unintended consequences of human activity, such as pollution), whereas the former is the practice of intentionally creating new devices and reshaping one's environment. Yet, the study of natural systems, especially biological ones, and the production of artificial systems could share much more than they currently do. On the one hand, biology provides an exceptional source of inspiration for future and emerging technologies (biotech, nanotech, cognitive science). Understanding cells and organisms by theoretical reconstruction, including "unrealistic computer models" (Bedau 1999), can give rise to a new generation of artificial systems endowed with an autonomy and adaptivity unmatched in classical engineering. On the other hand, closing the loop, these new algorithmic principles can be reimplemented in biological matter to create a novel type of physical-informational hybrid systems.

This article explores the recent move toward a "computationalization" and "physicalization" of theoretical biology, fast becoming reality under the aegis of bioinspiration and biomimetics. Within this general trend, we focus on the notion of architecture and the arduous double undertaking that it entails: instill more self-organization into computing artefacts (swarm robotics) and, conversely, instill more information technology into self-organizing natural objects (synthetic biology). The foundational thesis of this paper is that we can find a solution, both conceptual and operational, to the apparent paradox raised by the challenge of "programming self-organization". By identifying morphogenetically architected complex systems (MACS) to be those systems composed of a large set of elements that collectively and reliably produce specific structures from local interactions only, we can conceptually take emergent properties seriously, while operationally not give up on designing such systems.

\section{Morphogenetically Architected Complex Systems (MACS)}

First, we claim that MACS are based on specific emergent processes creating functional properties. A key aspect is the foundation of this category of systems on the concept of architecture.

\subsection{Definitions}

We start with a few conceptual definitions. A system is a durable entity comprising a group of elements, which may not themselves be durable. Systems can be living or inanimate, purely natural (no human intervention), natural-social (caused by humans without grand plan: cities, markets, Internet), purely artificial (machines), or hybrid artificial-natural (biotechnologies). The structure of the system denotes the particular arrangement of its elements, while its behavior results from what they do together and in interaction with the environment, where they can trigger external reactions called effects. When focusing on structure, the emphasis is put on the role of parts played by the elements: the system is composed of parts, which in most cases have 
specific spatial locations, but not always (website, ecosystem).

The form is the measurable geometric features that the system possesses in relation with its structure. Although there can be structure without form, most biological and engineered systems exhibit a lasting and recognizable form. In all types of systems, living or artificial, the form is created by a process or series of processes called morphogenesis. When the interactions among its elements, and between its elements and the environment, lead to structures or forms that are not easily predictable from the sole knowledge of the elements, the system is said to be complex. An architected system is not any structured system. Its architecture is not only an orderly arrangement of the elements of the systems (an ordered structure) but, more precisely, an orderly arrangement of its parts. This order relation specific to architecture comes from hierarchical relationships between parts and subparts, via groups and subgroups. Certain parts can be structurally more important if they are supporting, directly or indirectly, other parts-for instance, in construction, preventing the overall architecture from collapsing (keystones, hubs). Other parts can be more important from a maintenance viewpoint, etc. Hence, an architected system is fundamentally heterogeneous, as different parts contribute differently to its form and viability.

Just like a structure can lack a form, a system can also have a form without a clear architecture. Typically, a pattern is any repetitive element of the form of a system. As such, a pattern means that parts of the structure of the systems are equivalent, i.e. interchangeable, from the standpoint of its form. Although a pattern can itself display some internal symmetry or dynamical equilibrium, it does not reveal any particular order between parts. It is an element of the form that, in itself, is not indicative of any architecture, even if it can often be a part of one. An organized system is a system whose structure suggests symmetry, equilibrium, harmony, coherence, meaning, or function. It is one that seems to be organized to some end, first and foremost its own morphogenesis and self-maintenance, namely through self-organization. A selforganized system increases its statistical complexity while at the same time giving birth to emergence that makes its complexity drop relative to a new level of perception (Shalizi 2006). For us, MACS are those self-organized systems whose emerging properties are appealing mainly in the sense that the new perception level that they trigger often reveals the types of function that the system can have. Godfrey-Smith (2004, p286) proposes that for a living system, "the function of a structure is the effect it has that has been responsible for its being selected for" (our emphasis). Accordingly, and more broadly, we propose to define a function of the structure of any system (living or not, natural or not) as any effect it has that has been responsible for its being selected, modified or designed. In the following, we employ "function of the system" or "functional property" to denote a function of the structure.

\subsection{MACS and Their Specific Emergent Properties}

Artificial Life (ALife), an original research field integrating computational biology with bioinspired computing, studies in particular the properties of self-organization in complex systems. According to its principal founder, Chris Langton (1989), ALife "can contribute to theoretical biology by locating life-as-we-know-it within the larger picture of life-as-it-could-be". The main mechanisms of self-organization include 
negative and positive feedback, acquisition of information, and stigmergy (coordinated actions based on traces left in the environment, essentially used by social insects). Self-organization is the core engine of the dynamics of complex systems and their structure. In particular, it gives rise to a whole class of form-creating processesglobally subsumed under "morphogenesis".

Yet, a clear distinction can be drawn between two major types of form-creating complex systems: ones that display simple repetitive patterns (spots and stripes) or fractal motifs, and ones that produce sophisticated functional forms (bodies and constructions). Whereas the former are essentially stochastic and statistically homogeneous, the latter are noticeable for their "strong" morphogenetic properties, in that they demonstrate the ability to combine self-organization with architecture (Doursat et al 2013). At the time of his famous paper "The chemical basis of morphogenesis", Alan Turing (1952) was well aware of the qualitative distinction between pattern formation and morphogenesis, as he is said to have quipped: "The stripes are easy, it's the horse part that troubles me". For Philip Ball (1999), there is always an element of subjectivity in the perception of patterns, but one can distinguish simple shapes by the regular repetition of a unit, whereas an architected form has an individual character.

We are interested here in the second category, since they represent decentralized systems endowed with a hierarchical architecture, which can be observed in biology at every scale (cells, organisms, collective constructions) and also in certain engineered devices of a highly distributed nature (multi-agent software). This is for example the case of embryogenesis, through the self-assembly of cell masses creating a detailed and hierarchical anatomy; of insect colonies, through swarm collaboration building sophisticated constructions; and of the brain, through the myriads of neural signals synchronizing into ordered cognitive states. The distinctive characteristic of these complex systems is to give birth to "interesting" (i.e. nontrivial) emergent properties as they cause the generation of a specific, heterogeneous, and hierarchical form. In this sense, they implement a truly morphogenetic process.

To describe these "architectures without architects", we use the concept of MACS introduced above. It allows elaborating upon the aforementioned similarities between spontaneously evolved biological complex systems and human-caused industrial complex systems, while at the same time, precisely, putting a new focus on their common architectural aspects. MACS are specific among the systems traditionally studied in ALife in that they essentially give rise to emergent properties taking the form of emergent architectures. In sum, a MACS is a complex system 1) that has a form; 2) whose form possesses not only patterns but an architecture caused by its morphogenetic processes; 3) whose complexity at least partly resides in this architectural form, i.e. makes it difficult to predict from the sole properties of the elements. From there, we address the question of (re)taking control of these systems, i.e. guiding or programming them toward specific and beneficial outcomes. Following what the three cases above (embryogenesis, insect colonies, the brain) seem to indicate, we will now assume that many of the specific functional properties of living systems depend on their structure being not only self-organized but also self-architected. This architecture itself is the result of complex morphogenetic processes that are not entirely out of reach, neither from a cognitive nor from an operational standpoint. 


\section{Emergence in MACS}

At this point, an objection could be that, with MACS, we are facing a kind of "strong emergence", therefore one could not contemplate their controllability, let alone programmability. In this section, we take a closer look at the notion of "emergence" and argue that, in fact, to be a MACS a system does not need to display more than weak emergent properties. As a consequence, steering or programming the emergent properties of MACS becomes possible.

\subsection{To Deduce or to Compute}

It seems paradoxical to advocate any "programming of emergence", in particular to propose to modify or create spontaneous form-creating complex systems using computing and engineering concepts and tools. If one "programs" a process, it seems that nothing could possibly "emerge" from it. Yet, by considering emergence in its broadest meaning of a result or an "outcome", this apparent paradox is actually a fundamental and rather common trait of all mathematical or computational works. On the one hand, most of the classical mathematical theories of biological morphogenesis, such as D'Arcy Thompson's structuralism, Alan Turing's reaction-diffusion model (Turing 1952; Petitot 2013) or René Thom's catastrophe theory (Thom 1994; Petitot 2011) are founded on sets of axioms that show, through deductive reasoning, how a global level of patterns and forms that did not exist initially, therefore was not described at the local level, can ultimately arise.

On the other hand, although ALife is also committed to a characterization of living processes in the most general terms, this new field really took on when it became increasingly clear that computational emergence in the modeling and simulation of non-linear biological processes could not always be subsumed under mathematical emergence. As Thompson (2006, p305) writes: "Non-linear systems, in principle, are such that the set of equations defining the system do not permit a deduction of the future states of the system. The most common method of dealing with this fact is the use of computer simulation in what are termed numerical experiments". Hence, ALife is a necessary complementary approach to theoretical biology because axiomaticdeductive frameworks, which operate by transformations of symbolic strings, are not congruent to rule-based, algorithmic computing (Dowek 2011).

\subsection{Weak Emergence}

Philosophical positions and debates on the correlated notions of emergence and complex systems are numerous (Humphreys 1997; Kim 1999; Petitot 2003; Huneman 2008a; Brodu 2009; Ladyman et al 2013; Sartenaer 2014). Still, there is broad agreement that emergent phenomena are simultaneously (1) dependent on underlying processes and (2) autonomous from them-despite the vagueness of these terms. We follow here the distinctions made by Bedau $(1997,2002)$ as they are robust, operational in complex systems research and apply especially well to ALife. There is nominal emergence when these two hallmarks are observed, and the emergent property is different but predictable and explainable from the properties of the components (e.g. a 
circle and the equidistance of its points from a center). This "derivation" is not a computation but a mathematical deduction based on axioms and reasoning. Strong emergence or "downward causation" is invoked when the emergent property has a causal power in itself which is in no way reducible to the causal powers at the micro level (Kim 1999). However, it leads to contradictory cases of overdetermination, where micro states can be determined both upward by micro interactions (hallmark 1), and downward by macro actions. Consequently, "there is no evidence that strong emergence plays any role in contemporary science" (Bedau 2002, p11). Somewhere in the middle is weak emergence, or computational emergence (Huneman 2008b), applying to emergent properties of a system $\mathrm{S}$ that cannot be derived except by simulation. It can be defined by introducing entities and properties on two levels, micro and macro (Bedau 1997). "The state of a micro entity consists of its location and its possession of intrinsic properties, and its state changes if these change. A macro entity also has a state, and this consists simply in the aggregation of the states of all its component micro entities and their spatial relations." (Bedau 2002, p14). The causal dynamics at the micro level is captured by explicit state transition rules in every micro entity.

With this, a nominally emergent macro property $\mathrm{P}$ of $\mathrm{S}$ "is weakly emergent iff $\mathrm{P}$ is derivable from all of S's micro facts [dynamics, states, locations] but only by simulation" ( $p 15)$. This is an objectivist definition of emergence to the extent that it is based at least on computational incompressibility. Huneman (2008b) follows this line but advocates that only the computational process should be called emergent, not its resulting properties. This shift allows excluding purely random, patternless outcomes from the class of emergent properties. A subjectivist extension of this minimal objectivist account was suggested by Varenne $(2009,2013)$ through a further analysis of the notion of "simulation". He argues that different types of weak emergence can be found depending on the type of computational simulation (numerical, rulebased, agent-based). In the present study, Bedau's characterization will be shown to be sufficient when used for experimenting on (i.e. analyzing, reconstructing or reengineering) weak emergent phenomena via simulated or material systems.

To be sure, ALife has taken on in many ways and on many dimensions the grand challenge addressed here: the simulation or replication of complex systems so as to control or exploit emergence. Surprisingly, however, it has also often eschewed the specific import of computational emergence in its various models and creations. The reason might be that bioinspired disciplines, such as gene-inspired evolutionary computation, ant-inspired swarm intelligence, and neuron-inspired machine learning, needed to establish themselves upon solid mathematical foundations, hence were ineluctably pulled toward deductive frameworks, i.e. nominal emergence. Today, they are mainly concerned with solving blackbox problems (parametric estimation, combinatorics, classification, clustering) by metaheuristic and stochastic optimization methods, which brings them much closer to statistics than theoretical biology.

\subsection{Weak Emergence in MACS: Examples}

Our assertion is not that all emergent properties of all MACS are or can be seen as the result of a weak emergent process. What we want to show is that many emergent properties of many MACS, whether natural, artificial or hybrid, can be captured, un- 
derstood, simulated, replicated, and possibly enhanced or even redesigned, from the sole perspective of weak emergence with appropriate artificial computational devices.

Functional forms are most striking in the bodies of biological organisms, but not unique to them. They can also be artificial or produced by living agents from inert materials. Thus a machine like a car engine has a functional form, and so do emergent structures such as the nests built by colonies of social insects. The hexagonal cells of wasp nests or bee hives are emergent self-architected parts of the superorganism composed of the insects and their construction (Turner 2000), which are more than just patterns since they also serve as incubators for the larvae. The spots on a leopard or the triangles on a sea shell, however, cannot be qualified as functional forms strictly speaking, in that the functional consequences of these forms are not immediate. They do not play a key role in either the morphogenetic process or the viability of the organism, except for a long-term evolutionary advantage (camouflage, sexual attraction, anti-predator warning).

Functional forms can be seen as subparts of the system, also referred to as "bricks" (Holland 2012) or "functional blueprints" (Beal 2011). One of the most glaring differences between complex adaptive systems (such as termites and their mound) and most, although not all, cellular automata with homogeneous rules, is that the former can generate architectures, whereas the latter generally exhibit patterns only. The appearance of functional forms equips the system with a structure-specifically, a functional architecture that is a much more heterogeneous than repetitive. Next, we examine three major examples of MACS: proteins, multicellular development and insect constructions.

Proteins A first example are proteins, omnipresent in living systems, which are unidimensional chains of amino acids when synthesized, then quickly fold upon themselves at multiple scales in intricate but very specific and reproducible ways, giving it a complicated 3D architecture called the reference conformation. Therefore, a protein is a complex self-architected systems endowed with a functional form in that it is related to a particular function which operates directly: for example, it makes an antibody capable of binding to a particular pathogen, or accelerates the production of certain metabolites through enzymatic reactions. To paraphrase again Godfrey-Smith (2004, p286), it means that the function given by the protein's architecture is the effect it has that has been responsible for its being selected for. Protein folding has been a famous problem for over half a century, approached by various methods from physics and computer science, which have given rise to successful, if costly, molecular dynamics simulations (Dill and MacCallum 2012).

Multicellular development During embryogenesis, collective movements emerge from individual biomechanical behaviors via intercellular communication and coordination over long distances (relying on chemical mediators, electric tranmission or mechanotransduction). No single cell plays the role of a leader that governs an entire process. While biologists produce and annotate time-lapse microscopy of organism development, mathematicians and computer scientists need to process these images to reconstruct and model the collective cell dynamics. A collaborative effort of this kind has resulted in a software platform, BioEmergences (Peyriéras 2011), which provides 
automated analysis and reconstruction of collective cell movements. It can automatically handle large amounts of 4D (3D+time) voxel-based movies through a workflow of detection, segmentation and tracking algorithms. The main output is a "digital embryo" represented by a cell lineage tree annotated with quantitative measurements of nucleus positions and membrane shapes (Castro-González et al 2014). In parallel, one can attempt agent-based modeling and simulation centered on the physicochemical coupling of cell mechanics with gene expression and molecular signaling (Delile et al 2013). Cells' mechanical properties (division, adhesion, motility) are coupled with their spatial location and temporal state of gene regulation and molecular dynamics (protein and ligand concentrations), affecting each other concurrently. Exploration of parameter space is supported by reconstructed data, which allows measuring the "fitness" of the virtual embryo and validating hypotheses. The necessity to reconstruct the whole history of the first phases of the embryo via computer-based simulation shows that weak emergence is taken seriously from a cognitive viewpoint and made operational, too.

Social insect constructions In Africa, harvester termites build "cathedrals" that are the largest and most sophisticated of animal constructions (Camazine et al 2003). On the exterior, termite mound looks like a rough cone covered in ridges and stacks. The walls, made of earth pellets, enclose ventilation ducts running along the entire height. In the interior, empty spaces let air circulate around the central nest, and rooms surrounding fungi gardens contain chewed wood chips that serves as a substrate to crops. Below are the nurseries, made of thin strips supported by pillars, and in the center a bunker with thicker walls, the royal chamber, just large enough to contain the queen. Therefore, like multicellularity at a higher scale (Turner 2000), a termite mound is emblematic of a self-organized, unplanned architecture, i.e. a MACS. What is also fascinating is how well it meets the needs of the colony. Despite a relative lack of data to explain the formation of the most complicated structures, mathematical and computational models (Bonabeau et al 1998; Camazine et al 2003) can account for parts of them and extrapolate to the rest, based on stigmergic principles. Most social insects secrete pheromones, which are chemical substances triggering particular reactions in the individuals of the same species. The environment where pheromones are deposited and other physical clues are left, such as the current state of construction, becomes the support for an indirect stimulus/response communication among building agents. Consequently, these complicated and directly functional architectures can be simulated, understood and replicated as typically weak emergent systems, too.

\section{Controlling and Programming Weak Emergence in MACS}

Finally, since weak emergence is itself based on the possibility to simulate emergence, computationally or materially, we show that the notion of MACS is a good candidate to describe interesting and common properties of both living and artificial self-architected systems. By their focus on architecture, MACS transcend to some extent the dividing line between biology and engineering, conceptually and operationally, leading to concrete means of controlling and programming this emergence.

But how can a functional form be both wanted and emergent? How can we 
build an emergent architecture? We now address the question of (re)taking control of MACS, i.e. guiding or programming them toward specific and beneficial outcomes. Broadly, this can be achieved in two ways: by instilling more self-organization into those artefacts that compute via an architecture and, conversely, by instilling more information technology into self-architected natural objects. The first approach is illustrated below by morphogenetic collective robotics; the second by morphogenetic synthetic biology. Both are instances of morphogenetic engineering, a new field examined in the discussion.

\subsection{Morphogenetic Collective Robotics}

The field of collective robotics is about building agents that are embodied (with physical dimensions, not pure software), located (interacting in a changing environment through sensors/actuators), autonomous (without external guidance) and distributed (operating together decentrally) (Kube and Zhang 1993). These are tasks that classical artificial intelligence has proven unable to program robustly or only with great difficulty. Holland distinguishes between two broad classes of behavior: moving through and acting on the environment. All have already been performed by multi-robot systems: for example, flocking, collision avoidance, and scouting for the first; pushing heavy objects and sorting waste for the other. MACS engineering requires a system capable of the latter, but also of producing structures and morphologies adapted to different tasks. A system like Swarmorph (O'Grady et al 2009) provides a good template to explore such self-assembly capabilities. Given distributed and situated agents, the main challenge is to cross an arena littered with obstacles that cannot be overcome by a single agent but only by adequate group formations and morphologies. Experiments were conducted in simulation and actual conditions on the Swarmbot or (s-bot) robotic platform. By attaching to each other, s-bots are able to cross a trench in single file, or pass over a bridge in pairs using lateral connections. The two essential properties is that they do this without external control and without prior knowledge of the order in which they encounter obstacles. To improve their reactivity, obstacles can be analyzed from different viewpoints using a multi-species system such as Swarmanoid (Dorigo et al 2013), in which walking "foot-bots", climbing "hand-bots" and flying "eye-bots" communicate and exchange information. All these examples show that physical implementation of complex self-architected systems can operationally supplement, without conceptually overcoming it, a purely computer-based analysis of weak-emergent phenomena.

\subsection{Morphogenetic Synthetic Biology}

Beyond bioinformatics, synthetic biology can also be construed as a systems design challenge, akin to large software systems and electronic circuits. This is the position taken by the SynBioTIC project (Pascalie et al 2015), which is positioned upstream at the cell population level. From the "wetware" viewpoint, its motivation is to exploit the nontrivial collective properties of bacteria. To this aim, SynBioTIC proposes to design and develop formalisms and computer tools to translate the desired overall behavior of a population of cells into processes local to each cell. It relies on the spec- 
ification of a global spatial behavior and its description across a "tower of languages". Each language at a given level addresses distinct features. Its set of instructions can be literally "compiled" into the lower level, and ultimately down to the bioware into a cellular regulation network (gene regulatory network, signaling and metabolic pathways). This soft-to-wet approach, similar to a classical soft-to-hard compiler, aims to fill the gap between the high-level description of a biosystem and its low-level physical requirements, betting on their "reemergence". From the software viewpoint, this project relies on spatially explicit bacterial modeling and abstract "spatial/amorphous computing" frameworks, such as the MGS language (Giavitto and Michel 2002), to deal with new classes of applications characterized by the weak emergence of a global behavior in a large population of cells irregularly located and dynamically interacting. Naturally, the complexity of the design task increases tremendously when targeting elaborate shapes. Faced with a huge number of possible rules and parameters, rational design becomes untenable and one must resort to evolutionary computation. Yet, because real-world evolution is not driven by final cause, evolutionary metaheuristics should not run unbridled but rather be steered by some amount of rational intervention from a human designer, in this case via a formal language-tower architecture.

\section{Discussion}

\subsection{Morphogenetic Engineering}

Thus we are witnessing the emergence of a new discipline exploring the artificial design and implementation of systems that are both self-organized and able to develop sophisticated and reproducible architectures. To this extent, the study of MACS has its origins in Morphogenetic Engineering (ME), a field recently founded by Doursat, Sayama, and Michel $(2009,2013)$. Rather than direct methods of "top-down" design, i.e. implementing a function along predictable and preconceived articulations of parts, ME focuses on meta-design (Doursat 2008), i.e. the generic conceptual and operational rules at the level of the elements, needed for the endogenous self-assembly, self-regulation and evolution of an artificial system, drawing from processes seen in natural MACS. "Don't build a system directly, but shape its building blocks in such a way that they do it for you" (Doursat et al 2013, p520). Thus it is about guiding, rather than planning, the growth and formation of structures within a community of agents. How do biological organisms and populations perform morphogenetic tasks reliably? Can we export their precise self-formation capabilities to engineered systems?

Application fields are numerous and, beside self-assembling robots, may involve self-distributed software programming, self-reconfiguring production lines or selfstabilizing energy grids. Four categories of ME systems can be identified, according to the type of macro phenomena that they embody (Doursat et al 2013): constructing (components attach into "stick-figures"), coalescing (many agents swarm into shapes), developing (agent mass grows by division or aggregation; Doursat and Sánchez 2014), generating (agents are inserted by a "grammar"). On the micro level, Sayama (2014) defines a perpendicular axis of ME taxonomy through four increasingly elaborate types of agents: homogeneous (agents are stateless; function of observations only), heterogeneous (agents are stateful), differentiating (agent states can dy- 
namically change), and communicating (agents share information locally). He shows that while heterogeneity has a strong impact on the structure and behavior of the swarms, dynamic differentiation and local information sharing also help them maintain a spatially adjacent, coherent organization.

\subsection{A Possible Objection and Conclusion}

We claimed earlier that few ALife research works actually addressed or leveraged the full power of emergence, despite the fact that all biological systems are complex by nature. We suggested that if the main goal was to understand and accelerate the growing convergence between biology and engineering, then one could no longer avoid confronting emergent properties in simulated, designed or hybrid systems. Now, it could be objected that this suggestion remains vague, hence questionable, because, as we have also shown, not all emergent properties are interesting but only those properties that are immediately functional for the system. In fact, this is where conceptual difficulties arise. There exists some agreement in the literature about certain quantifiable aspects or measures of emergence in complex systems, specifically around the concept of "statistical complexity" after the work of Crutchfield (1994) and Shalizi (2006). In summary, "statistical complexity measures the amount of information that is present in the past of a system which is relevant to predict its future" (Brodu 2009, p41). But function, especially in a biological context, is the kind of property that is often difficult to capture by a quantifiable measure. From this perspective, functional emergence is very close to what Pattee (1995) calls "semantic emergence". Whereas "syntactic emergence refers to how an entity defined at a higher level of investigation appears in the lower level...semantic emergence is when some function of the entity may not be described within the formal lower level system" (Brodu 2009, p41).

Similarly Godfrey-Smith (2004, p287) recalls that "A number of philosophers have thought that there is a very close relation between functional properties, in this special evolutionary sense [which we have adopted here], and semantic properties", citing Millikan (1984) on this topic. The meaning of sentences and words typically is of the latter type. Therefore, it cannot be directly expressed via physical and architectural properties, or the intrinsic aspect of the "weak" type of emergence, which was in the foreground of MACS. The answer to this objection lies in the correlative emphasis that we constantly put on the essential spatial and architectural dimensions of the systems under study: not function alone, but functional form. The concept of MACS intentionally stresses "architecture", then "form". In all the examples that we gave, functional emergence can also be interpreted in terms of weak emergence because the property at the higher level can still be meaningfully described and measured in terms of forms, places, locations, movements-specifically as the construction of chambers or walls (termites), the assembly of rows (Swarmorph), and so on. This is the reason why some type of quantification, then engineering and control, can be contemplated for this specific kind of functional emergence.

This final aspect confirms our main conclusion that adopting the not overly general but operational and transdisciplinary concept of MACS can help cross-fertilize theoretical and experimental approaches to complex systems (especially living ones) that try to conceive and control them relatively to their functions. 


\section{References}

Ball P (1999) The Self-Made Tapestry: Pattern Formation in Nature. Oxford Univ Press

Beal J (2011) Functional blueprints: An approach to modularity in grown systems. Swarm Intell 5:257-281

Bedau MA (1997) Weak emergence. Noûs 31:375-399

Bedau MA (1999) Can unrealistic computer models illuminate theoretical biology? In: GECCO, pp 20-23

Bedau MA (2002) Downward causation and the autonomy of weak emergence. Principia 6:5-50

Bonabeau E, et al (1998) A model for the emergence of pillars, walls and royal chambers in termite nests. Philos T R Soc B 353:1561-1576

Brodu N (2009) A synthesis and a practical approach to complex systems. Complexity 15:36-60

Camazine S, et al (2003) Self-Organization in Biological Systems. Princeton Univ Press

Castro-González C, et al (2014) A digital framework to build, visualize and analyze a gene expression atlas with cellular resolution in zebrafish early embryogenesis. PLoS Comp Biol 10:e1003,670

Crutchfield JP (1994) The calculi of emergence: Computation, dynamics \& induction. Physica D 75:11-54

Delile J, Doursat R, Peyriéras N (2013) Computational modeling and simulation of animal early embryogenesis with the MecaGen platform. In: Computational Systems Biology 2, Acad Press, pp 359-405

Dill KA, MacCallum JL (2012) The protein-folding problem, 50 years on. Science 338(6110):1042-1046 Dorigo M, et al (2013) Swarmanoid. IEEE Robot Autom Mag 20:60-71

Doursat R (2008) Organically grown architectures. In: Organic Computing, Springer, pp 167-199

Doursat R, Sánchez C (2014) Growing fine-grained multicellular robots. Soft Robotics 1:110-121

Doursat R, Sayama H, Michel O (2009) 1st Int Morphogenetic Eng Workshop. http://iscpif.fr/mew2009

Doursat R, Sayama H, Michel O (2013) A review of morphogenetic engineering. Nat Comp 12:517-535

Dowek G (2011) Proofs and Algorithms: An Introduction to Logic and Computability. Springer

Giavitto JL, Michel O (2002) The topological structures of membrane computing. Fund Info 49:123-145

Godfrey-Smith P (2004) Genes do not encode information for phenotypic traits. In: Contemporary Debates in Philosophy of Science, Blackwell Publishing, pp 275-289

Holland JH (2012) Signals and Boundaries: Building Blocks for Complex Adaptive Systems. MIT Press

Humphreys P (1997) How properties emerge. Philos Sci pp 1-17

Huneman P (2008a) Emergence and adaptation. Minds and Machines 18:493-520

Huneman P (2008b) Emergence made ontological? Philos Sci 75:595-607

Kim J (1999) Making sense of emergence. Philos Stud 95:3-36

Kube CR, Zhang H (1993) Collective robotics: From social insects to robots. Adapt Behav 2:189-218

Ladyman J, Lambert J, Wiesner K (2013) What is a complex system? Eur J Philos Sci 3:33-67

Langton CG (1989) Artificial Life. Addison-Wesley

Millikan RG (1984) Language, thought and other biological categories. The MIT Press

O'Grady R, Christensen AL, Dorigo M (2009) Swarmorph. IEEE Trans Robot 25:738-743

Pascalie J, et al (2015) Spatial computing in synthetic bioware. In: Proc Europ Conf Artificial Life 2015

Pattee H (1995) Evolving self-reference: Matter, symbols and semantic closure. Commun Cognit 12:9-27

Petitot J (2003) Modèles de structures émergentes dans les systèmes complexes. In: Proc Annual Meeting Int Acad Philos Sci, pp 57-71

Petitot J (2011) The morphogenetic models of René Thom. In: Morphogenesis, Springer, pp 273-281

Petitot J (2013) Complexity and self-organization in Turing. In: The Legacy of A.M. Turing, Franco Angeli, Milano, pp 149-182

Peyriéras N (2011) Animal morphogenesis. In: Morphogenesis, Springer, pp 167-188

Sartenaer O (2014) Synchronic vs. diachronic emergence: a reappraisal. Eur J Philos Sci 5:31-54

Sayama H (2014) Four classes of morphogenetic collective systems. In: Artificial Life 14, pp 105-114

Shalizi CR (2006) Methods and techniques of complex systems science: An overview. In: Complex Systems Science in Biomedicine, Springer, pp 33-114

Thom R (1994) Structural Stability and Morphogenesis. Advanced Books Classics. Westview Press

Thompson P (2006) A role for mathematical models in formalizing self-organizing systems. In: SelfOrganization and Emergence in Life Sciences, Springer, pp 301-313

Turing AM (1952) The chemical basis of morphogenesis. Philos T R Soc B 237:37-72

Turner JS (2000) The extended organism: the physiology of animal-built structures. Harvard Univ Press

Varenne F (2009) Models and simulations in the historical emergence of the science of complexity. In: From System Complexity to Emergent Properties, Springer, pp 3-21

Varenne F (2013) Chains of reference in computer simulations. Tech. Rep. WP-2013-51, FMSH Working Paper, URL https://halshs.archives-ouvertes.fr/halshs-00870463 